\title{
ON A THEOREM OF DIXMIER
}

\author{
KUNG-FU NG
}

A well-known theorem of Alaoglu (cf. [3, p. 84]) tells us that the closed unit ball in the Banach dual space of a normed space is compact with respect to the $w^{*}$-topology. In [1], Dixmier showed that this property is characteristic for Banach dual spaces. In this note, we shall give a short proof of a variant of Dixmier's theorem. This variant appears to be more convenient for applications [2]. Our argument is inspired by Edwards' paper [2] and is strictly elementary (in particular, we do not use the Krein-Smulian theorem).

Theorem 1. Let $(X,\|\cdot\|)$ be a normed space with closed unit ball $\Sigma$. Suppose there exists a (Hausdorff) locally convex topology $\tau$ for $X$ such that $\Sigma$ is $\tau$-compact. Then $X$ itself is a Banach dual space, that is, there exists a Banach space $V$ such that $X$ is isometrically isomorphic to the dual space $V^{\prime}$ of $V$ (in particular, $X$ is complete).

Proof. Let $(X, \tau)^{\prime}$ and $(X,\|\cdot\|)^{\prime}$ denote the dual spaces of $X$ under $\tau$ and $\|\cdot\|$ respectively. Let $V$ be the space of all linear functionals $f$ on $X$ such that $f$ is $\tau$-continuous on $\Sigma$. Then

$$
(X, \tau)^{\prime} \cong V \cong(X,\|\cdot\|)^{\prime} .
$$

The first inequality is obvious, and to see the second, let $f \in V$. Then $f(\Sigma)$ is the continuous image of the $\tau$-compact set $\Sigma$, so is compact and hence bounded. Therefore $f$ is continuous on $(X,\|\cdot\|)$, and (1) is proved. Now it is easily seen that $V$ is a closed subspace of the Banach space $(X,\|\cdot\|)^{\prime}$. Thus, $V$ may be regarded as a Banach space in its own right.

For each $x$ in $X$, define $\varphi(x)$ by the rule

$$
(\varphi(x))(v)=v(x), \quad v \in V .
$$

Then it is easy to see that $\varphi$ is a 1-1 continuous (in fact norm-reducing) map from $X$ into the Banach dual space $V^{\prime}$ of $V$. Also, since each $v$ in $V$ is $\tau$-continuous on $\Sigma$, the restriction $\varphi \mid \Sigma$ of $\varphi$ to $\Sigma$ is continuous with respect to the relative $\tau$-topology and the $w^{*}$-topology $\sigma\left(V^{\prime}, V\right)$. Since 
$\Sigma$ is $\tau$-compact, it follows that $\varphi(\Sigma)$ is $\sigma\left(V^{\prime}, V\right)$-compact. Also, this set $\varphi(\Sigma)$ is convex. By the bipolar theorem (cf. $[3,126])$, it is precisely its bipolar $[\varphi(\Sigma)]^{n \pi}$ with respect to the duality $\left(V^{\prime}, V\right)$. Note that

$$
[\varphi(\Sigma)]^{\pi}=\{v \in V:(\varphi(x))(v) \leqq 1, \forall x \in \Sigma\}=\{v \in V: v(x) \leqq 1, \forall x \in \Sigma\},
$$

which is just the unit ball in $V$, and hence $[\varphi(\Sigma)]^{\pi n}$ (that is, $\varphi(\Sigma)$ ) is the unit ball in $V^{\prime}$. In other words, $\varphi$ maps $\Sigma$ onto the unit ball in $V^{\prime}$. Therefore $\varphi$ is an isometry and onto the space $V^{\prime}$. The proof of theorem 1 is thus completed.

This theorem implies immediately the theorem of Dixmier referred to at the beginning:

Theorem 2. Let $(X,\|\cdot\|)$ be a Banach space with closed unit ball $\Sigma$. Suppose there exists a total subspace $V$ of $(X,\|\cdot\|)^{\prime}$ such that $\Sigma$ is $\sigma(X, V)$ compact. Then $X$ itself is a Banach dual space.

\section{REFERENCES}

1. J. Dixmier, Sur un théorème de Banach, Duke Math. J. 15 (1948), 1057-71.

2. D. A. Edwards, On the homeomorphic affine embedding of a locally compact cone into a Banach dual space endowed with the vague topology, Proc. London Math. Soc. (3), 14 (1964), 399-414.

3. H. H. Schaefer, Topological vector spaces, Macmillan, New York, 1966.

UNITED COLLEGE, THE CHINESE UNIVERSITY OF HONG KONG, HONG KONG 